\title{
TENSOR ABSOLUTE VALUE EQUATIONS
}

\author{
SHOUQIANG DU*, LIPING ZHANG ${ }^{\dagger}$, CHIYU CHEN ${ }^{\ddagger}$, AND LIQUN QI§
}

\begin{abstract}
This paper is concerned with solving some structured multi-linear systems, which are called tensor absolute value equations. This kind of absolute value equations is closely related to tensor complementarity problems and is a generalization of the well-known absolute value equations in the matrix case. We prove that tensor absolute value equations are equivalent to some special structured tensor complementary problems. Some sufficient conditions are given to guarantee the existence of solutions for tensor absolute value equations. We also propose a Levenberg-Marquardttype algorithm for solving some given tensor absolute value equations and preliminary numerical results are reported to indicate the efficiency of the proposed algorithm.
\end{abstract}

Key words. M-tensors, absolute value equations, Levenberg-Marquardt method, tensor complementarity problem

AMS subject classifications. 15A48, 15A69, 65K05, 90C30, 90C20

1. Introduction. The systems of multi-linear equations can be expressed by tensor-vector products, just as we rewrite linear systems by matrix-vector products. Let $\mathcal{A}$ be an $m$ th-order tensor in $R^{n} \times \cdots \times R^{n}$ and $\mathbf{b}$ be a vector in $R^{n}$. Then a multi-linear equation can be expressed as

$$
\mathcal{A} \mathbf{x}^{m-1}=\mathbf{b}
$$

where $\mathcal{A} \mathrm{x}^{m-1}$ is a vector in $R^{n}$ [21] with

$$
\left(\mathcal{A} \mathbf{x}^{m-1}\right)_{i}=\sum_{i_{2}=1}^{n} \cdots \sum_{i_{m}=1}^{n} a_{i i_{2} \ldots i_{m}} x_{i_{2}} \cdots x_{i_{m}}, \quad i=1, \ldots, n .
$$

Solving multi-linear systems is always an important problem in engineering and scientific computing [8, 15]. In this paper, we consider the systems of multi-linear absolute value equations, which can be expressed as

$$
\mathcal{A} \mathbf{x}^{m-1}-|\mathbf{x}|^{[m-1]}=\mathbf{b},
$$

where $|\mathbf{x}|^{[m-1]}$ is a vector in $R^{n}$ with

$$
|\mathbf{x}|^{[m-1]}=\left(\left|x_{1}\right|^{m-1}, \ldots,\left|x_{n}\right|^{m-1}\right)^{T} .
$$

It is easy to see that the system of multi-linear absolute value equations (1.2) is a generalization of the well-known absolute value equations

$$
A \mathbf{x}-|\mathbf{x}|=\mathbf{b}
$$

with a matrix $A \in R^{n \times n}$. The absolute value equations (AVE) has wide applications in applied science and technology such as optimization physical and economic

\footnotetext{
*College of Mathematics and statistic, Qingdao University, Qingdao 266071, P. R. China (dsq89330163.com).

$\dagger$ Corresponding author. Department of Mathematical Sciences, Tsinghua University, Beijing 100084, P. R. China (lzhang@math.tsinghua.edu.cn).

${ }^{\ddagger}$ Department of Mathematical Sciences, Tsinghua University, Beijing 100084, P. R. China (ccy_justin@163.com).

$\S$ Department of Applied Mathematics, The Hong Kong Polytechnic University, Hung Hom, Kowloon, Hong Kong, P. R. China (maqilq@polyu.edu.hk).
} 
equilibrium problems [17, 18, 19]. As was shown in [19, the general NP-hard linear complementarity problem [7] which subsumes many mathematical programming problems can be formulated as an AVE. This implies that the AVE is NP-hard in its general form. Analogous to AVE, we call (1.2) tensor absolute value equations (TAVE). Obviously, the TAVE is also NP-hard. Thus, investigating the existence of solutions for the TAVE is a significant problem.

Recently, Song and Qi 25] introduced a class of complementarity problems, called tensor complementarity problems, where the involved function is defined by some homogenous polynomial of degree $n$ with $n>2$. It is known that the tensor complementarity problem is a generalization of the linear complementarity problem [7; and a subclass of nonlinear complementarity problems [10. The tensor complementarity problem was studied recently by many scholars [4, 26]. In [19], it was shown that the $\mathrm{AVE}$ is equivalent to a generalized linear complementarity problem. Can we show that the TAVE is equivalent to a generalized tensor complementarity problem? Although some computational methods have been presented for the AVE, it is very difficult to extend these algorithms to solve the TAVE because the TAVE (1.2) is a nonlinear equation. The Levenberg-Marquardt method is one of the important algorithms for solving nonlinear equations [11. Can we propose an efficient algorithm such as the Levenberg-Marquardt method for solving the TAVE (1.2)? To our best knowledge, there is no general answer to these questions. Therefore, we shall focus on some special tensor absolute value equations.

Let $\mathcal{I}$ be an $m$ th-order $n$-dimensional unit tensor, whose entries are 1 if and only if $i_{1}=\cdots=i_{m}$ and otherwise zero. A tensor $\mathcal{A}$ is called a nonnegative tensor if all its entries are nonnegative, denoted $\mathcal{A} \geq 0$. A tensor is called a $Z$-tensor, if all its diagonal entries are nonnegative and off-diagonal entries are nonpositive. $M$-tensor is a special class of Z-tensor, which was first introduced and studied in [9, 29]. To define the $M$-tensors, we need to introduce the tensor eigenvalues first. Let $\mathcal{A}$ be an $m$ th-order $n$-dimensional tensor. If a scalar $\lambda \in R$ and a nonzero vector $\mathbf{x} \in R^{n}$ satisfy

$$
\mathcal{A} \mathbf{x}^{m-1}=\lambda \mathbf{x}^{[m-1]},
$$

where $\mathbf{x}^{[m-1]}=\left(x_{1}^{m-1}, \ldots, x_{n}^{m-1}\right)^{T}$, then we call $\lambda$ an eigenvalue of $\mathcal{A}$ and $x$ a corresponding eigenvector. Qi [21] and Lim [16] first defined the eigenvalues of tensors independently. The spectral radius of a tensor $\mathcal{A}$ is defined by

$$
\rho(\mathcal{A})=\max \{|\lambda|: \quad \lambda \text { is an eiegnvalue of } \mathcal{A}\} .
$$

A tensor $\mathcal{A}$ is called an $M$-tensor, if it can be written as $\mathcal{A}=s \mathcal{I}-\mathcal{B}$ with $\mathcal{B} \geq 0$ and $s \geq \rho(\mathcal{B})$; furthermore, it is called a strong $M$-tensor if $s>\rho(\mathcal{B})$. One can refer to a survey [3] for the spectral theory of nonnegative tensors. In this paper, we first investigate the existence of solutions for the TAVE (1.2). We show that the TAVE (1.2) with positive right-hand side $b$ always has a unique solution when $\mathcal{A}-\mathcal{I}$ is strong $M$-tensor. Another sufficient condition for the existence of solution is also given. Can we compute the solution? We propose an inexact Levenberg-Marquardt method for solving the TAVE (1.2).

The rest of this paper is organized as follows. In Section 2, we introduce the tensor absolute value equations which is a generalization of absolute value equations with matrix case. In Section 3, some sufficient conditions for the existence of solution to the TAVE are given. In Section 4, we first reformulate the TAVE as a special tensor 
complementarity problem and then we propose an an inexact Levenberg-Marquardttype algorithm for solving the TAVE. Some numerical results are reported in Section 5. Finally, some conclusions are given.

Throughout this paper, we assume that $m \geq 2$. We use small letters $\mathrm{x}, \mathrm{y}, \ldots$, for scalars, small bold letters $\mathbf{x}, \mathbf{y}, \ldots$, for vectors, capital letters $A, B, \ldots$, for matrixes, calligraphic letters $\mathcal{A}, \mathcal{B}, \ldots$, for tensors, calligraphic letters $\mathcal{D}$ for diagonal tensor whose diagonal elements are 1 or -1 . All the tensors discussed in this paper are real. $T(m, n)$ denotes the set of all $m$ th order $n$-dimensional tensors. Let $\mathcal{A}=\left(a_{i_{1} i_{2} \ldots i_{m}}\right) \in T(m, n)$, then $\mathcal{A}$ is called a symmetric tensor if its entries $a_{i_{1} i_{2} \ldots i_{m}}$ are invariant under any permutation of their indices. $S(m, n)$ denotes the set of all symmetric tensors. For such a matrix $A^{T}$ will denote the transpose of $A$. The identity matrix of arbitrary dimension will be denoted by $I$.

2. Tensor absolute value equations. In this section, we present some basic definitions and properties in absolute value equations, nonlinear complementarity problems, and nonsmooth analysis, which will be used in the sequel.

We recall the absolute value equations (AVE) of the type

$$
A \mathbf{x}-|\mathbf{x}|=\mathbf{b},
$$

where $A \in R^{n \times n}, \mathbf{b} \in R^{n}$ and $|\mathbf{x}|$ denotes the vector with absolute values of each component of $\mathbf{x}$. The AVE (2.1) has been widely investigated in many literatures such as [17, 18, 19. In [19], some results about the AVE are given, which we list as follows:

(i) The AVE (2.1) is equivalent to the bilinear program

$$
0=\min \left\{((A+I) \mathbf{x}-\mathbf{b})^{T}((A-I) \mathbf{x}-\mathbf{b}) \mid(A+I) \mathbf{x}-\mathbf{b} \geq \mathbf{0},(A-I) \mathbf{x}-\mathbf{b} \geq \mathbf{0}\right\},
$$

and the generalized linear complementarity problem

$$
(A+I) \mathbf{x}-\mathbf{b} \geq \mathbf{0}, \quad(A-I) \mathbf{x}-\mathbf{b} \geq \mathbf{0} \quad((A+I) \mathbf{x}-\mathbf{b})^{T}((A-I) \mathbf{x}-\mathbf{b})=0 .
$$

(ii) Let $C \in R^{n \times n}$ and $\mathbf{b} \in R^{n}$. Then

$$
(C-I) \mathbf{z}=\mathbf{b}, \quad \mathbf{z} \geq \mathbf{0} \quad \text { has a solution } \mathbf{z} \in R^{n}
$$

implies that

$$
A \mathbf{x}-|\mathbf{x}|=\mathbf{b} \quad \text { has a solution for any } A=C D \text { with } D=\operatorname{diag}( \pm 1) .
$$

Clearly, the tensor absolute value equation (1.2) is a generalization of the AVE (2.1) from the matrix case to the tensor case. Take an equation with the coefficient tensor $\mathcal{A} \in R^{2 \times 2 \times 2}$ as an example. The tensor absolute equation

$$
\mathcal{A} \mathbf{x}^{2}-|\mathbf{x}|^{2}=\mathbf{b}
$$

is a condense form of

$$
\left\{\begin{array}{l}
a_{111} x_{1}^{2}+\left(a_{112}+a_{121}\right) x_{1} x_{2}+a_{122} x_{2}^{2}-\left|x_{1}\right|^{2}=b_{1} \\
a_{211} x_{1}^{2}+\left(a_{212}+a_{221}\right) x_{1} x_{2}+a_{222} x_{2}^{2}-\left|x_{2}\right|^{2}=b_{2} .
\end{array}\right.
$$

We want to find $x_{1}$ and $x_{2}$ that satisfy the above two equations.

The following example shows a specific tensor absolute value equation. 
EXAMPLE 2.1. Let a tensor $\mathcal{A} \in T(4,2)$ be defined by $a_{1111}=a_{2111}=a_{2222}=1$, $a_{1222}=-1$, and zero otherwise. Let $\mathbf{b}=(1,2)^{T}$. Then the corresponding tensor absolute value equation is

$$
\left\{\begin{aligned}
x_{1}^{3}-x_{2}^{3}-\left|x_{1}\right|^{3} & =1 \\
-2 x_{1}^{3}+x_{2}^{3}-\left|x_{2}\right|^{3} & =2
\end{aligned}\right.
$$

By simplicity computation, we see that the TAVE (2.2) in Example 2.1 has no solution. In the next section we will discuss the existence of solution for the TAVE (1.2). We can extend the result (ii) to the TAVE and obtain a similar condition for the existence of solution to (1.2).

Below, we introduce the classical nonlinear complementarity problem. The tensor complementarity problem recently introduced in 25. is a special kind of nonlinear complementarity problem. It will be shown in Section 4 that the TAVE (1.2) can be reformulated as a special kind of generalized tensor complementarity problem.

Definition 2.1. Given a given mapping $F: R^{n} \rightarrow R^{n}$, the nonlinear complementarity problem, denoted by $\operatorname{NCP}(F)$, is to find a vector $\mathbf{x} \in R^{n}$ satisfying

$$
\mathbf{x} \geq \mathbf{0}, \quad F(\mathbf{x}) \geq \mathbf{0}, \quad \mathbf{x}^{T} F(\mathbf{x})=0 .
$$

Many solution methods developed for $\operatorname{NCP}(F)$ or related problems are based on reformulating them as a system of equations using so-called NCP-functions [10]. Here a function $\phi: R^{2} \rightarrow R$ is called an NCP-function if

$$
\phi(a, b)=0 \quad \Leftrightarrow \quad a \geq 0, b \geq 0, a b=0 .
$$

Given an NCP-function $\phi$, let us define

$$
\Phi(\mathbf{x})=\left(\phi\left(x_{1}, F_{1}(\mathbf{x})\right), \ldots, \phi\left(x_{n}, F_{n}(\mathbf{x})\right)\right)^{T} .
$$

It is obvious that $\mathbf{x} \in R^{n}$ is a solution of $\operatorname{NCP}(F)$ if and only if it solves the system of nonsmooth equations

$$
\Phi(\mathbf{x})=\mathbf{0} .
$$

For the solution of $\Phi(\mathbf{x})=\mathbf{0}$, we recall some definitions in nonsmooth analysis. Suppose that $\Theta: U \subseteq R^{n_{1}} \rightarrow R^{n_{2}}$ is a locally Lipschitz function, where $U$ is nonempty and open. By Rademacher's Theorem, $\Theta$ is differentiable almost everywhere. Let $D_{\Theta} \subseteq R^{n_{1}}$ denote the set of points at which $\Theta$ is differentiable. For any $\mathbf{x} \in D_{\Theta}$, we write $J \Theta(\mathbf{x})$ for the usual $n_{2} \times n_{1}$ Jacobian matrix of partial derivatives. The $B$-subdifferential of $\Theta$ at $\mathbf{x} \in U$ is the set defined by

$$
\partial_{B} \Theta(\mathbf{x})=\left\{V \in R^{n_{2} \times n_{1}} \mid \exists\left\{\mathbf{x}^{k}\right\} \subseteq D_{\Theta} \text { with } \mathbf{x}^{k} \rightarrow \mathbf{x}, J \Theta\left(\mathbf{x}^{k}\right) \rightarrow V\right\} .
$$

The Clarke's generalized Jacobian of $\Theta$ at $\mathbf{x}$ is the set defined by

$$
\partial \Theta(\mathbf{x})=\operatorname{co}\left(\partial_{B} \Theta(\mathbf{x})\right)
$$

where "co" denotes the convex hull. Then, $\partial \Theta(\mathbf{x})$ is a nonempty convex compact subset of $R^{n_{2} \times n_{1}}$ [6]. The function $\Theta$ is semismooth [13, 22] at $\mathbf{x} \in R^{n_{1}}$ if

$$
\lim _{\substack{V \in \partial \Theta(\mathbf{x}+t \tilde{\mathbf{d}}) \\ \tilde{\mathbf{d}} \rightarrow \mathbf{d}, t \downarrow 0}} V \tilde{d}
$$


exists for all $\mathbf{d} \in R^{n_{1}}$. If $\Theta$ is semismooth at all $\mathbf{x} \in U$, we call $\Theta$ semismooth on $U$. The function $\Theta$ is called strongly semismooth 23 if it is semismooth and for any $\mathbf{x} \in U$ and $V \in \partial \Theta(\mathbf{x}+t \mathbf{d})$,

$$
V d-\Theta^{\prime}(\mathbf{x} ; \mathbf{d})=O\left(\|\mathbf{d}\|^{2}\right), \quad \mathbf{d} \rightarrow \mathbf{0},
$$

where $\Theta^{\prime}(\mathbf{x} ; \mathbf{d})$ denotes the directional derivative [2] of $\Theta$ at $\mathbf{x}$ in direction $\mathbf{d}$, i.e.,

$$
\Theta^{\prime}(\mathbf{x} ; \mathbf{d})=\lim _{t \downarrow 0} \frac{\Theta(\mathbf{x}+t \mathbf{d})-\Theta(\mathbf{x})}{t} .
$$

Note that if the function $\Theta$ is semismooth at $\mathbf{x}$, the directional derivative $\Theta^{\prime}(\mathbf{x} ; \mathbf{d})$ exists for all $\mathbf{d} \in R^{n_{1}}$ and

$$
\Theta^{\prime}(\mathbf{x} ; \mathbf{d})=\lim _{\substack{V \in \partial \Theta(\mathbf{x}+t \tilde{\mathbf{d}}) \\ \tilde{\mathbf{d}} \rightarrow \mathbf{d}, t \downarrow 0}} V \tilde{d} .
$$

We now present some NCP-functions which are widely used in nonlinear complementarity problems. For more details about NCP-functions and their smoothing approximations, one can refer to [24, 30] and references therein.

Here we give some well-known NCP-functions as follows:

- The min function:

$$
\phi(a, b)=\min \{a, b\} .
$$

- The Fischer-Burmeister function:

$$
\phi_{F B}(a, b)=a+b-\sqrt{a^{2}+b^{2}} .
$$

It has been shown that all these NCP-functions are globally Lipschitz continuous, directionally differentiable, and strongly semismooth [12, 27. For example, the generalized gradient $\partial \phi_{F B}(a, b)$ of $\phi_{F B}(a, b)$ is equal to the set of all $\left(v_{a}, v_{b}\right)$ such that

$$
\left(v_{a}, v_{b}\right)= \begin{cases}\left(1-\frac{a}{\sqrt{a^{2}+b^{2}}}, 1-\frac{b}{\sqrt{a^{2}+b^{2}}}\right) & \text { if }(a, b) \neq(0,0), \\ (1-\xi, 1-\varsigma) & \text { if }(a, b)=(0,0),\end{cases}
$$

where $(\xi, \varsigma)$ is any vector satisfying $\xi^{2}+\varsigma^{2} \leq 1$.

In Section 4, we will use the Fischer-Burmeister function to reformulate the TAVE (1.2) as a system of equations and then we will propose an algorithm to solve the system of equations.

We now introduce the tensor complementarity problem which first defined by Song and Qi 25.

DEFINITION 2.2. Given any given tensor $\mathcal{A} \in T(m, n)$ and vector $\mathbf{q} \in R^{n}$, the tensor complementarity problem, denoted by $T C P(\mathcal{A}, \mathbf{q})$, is to find a vector $\mathbf{x} \in R^{n}$ satisfying

$$
\mathbf{x} \geq \mathbf{0}, \quad \mathcal{A} \mathbf{x}^{m-1}+\mathbf{q} \geq \mathbf{0}, \quad \mathbf{x}^{T}\left(\mathcal{A} \mathbf{x}^{m-1}+\mathbf{q}\right)=0 .
$$

Note that when $n=2$, the tensor $\mathcal{A}$ reduces to a matrix, denoted by $A$, and the $\operatorname{TCP}(\mathcal{A}, \mathbf{q})$ becomes: find a vector $\mathbf{x} \in R^{n}$ such that

$$
\mathbf{x} \geq \mathbf{0}, \quad A \mathbf{x}+\mathbf{q} \geq \mathbf{0}, \quad \mathbf{x}^{T}(A \mathbf{x}+\mathbf{q})=0,
$$


which is just the linear complementarity problem [7]. Very recently, a class of $n$-person noncooperative games are in [14, where the utility function of every player is given by a homogeneous polynomial defined by the payoff tensor of that player, which is a natural extension of the bimatrix game where the utility function of every player is given by a quadratic form defined by the payoff matrix of that player. Such a problem is called the multilinear game. The multilinear game is reformulated as a tensor complementarity problem. Some semismooth Newton-type methods are recently proposed for solving the tensor complementarity problems (see, e.g., [5]). In Section 4, we will extend the result (i) to the TAVE (1.2) and show that the TAVE (1.2) is equivalent to a bi-multilinear program and a generalized tensor complementarity problem.

3. Existence of solutions. In this section, we give some sufficient conditions for the existence of solutions to the TAVE (1.2). Specially, we extend the result (ii) about the AVE (2.1) to the TAVE (1.2).

We need the following lemmas which are recently established in [8, Theorems 3.2, $3.3,3.4]$.

Lemma 3.1. Let $\mathcal{A} \in T(m, n)$. If $\mathcal{A}$ is a strong $M$-tensor, then for every positive vector $\mathbf{b}$ the multilinear system of equations $\mathcal{A} \mathbf{x}^{m-1}=\mathbf{b}$ has a unique positive solution.

Lemma 3.2. Let $\mathcal{A} \in T(m, n)$ be a $Z$-tensor. Then it is a strong $M$-tensor if and only if the multilinear system of equations $\mathcal{A} \mathbf{x}^{m-1}=\mathbf{b}$ has a unique positive solution for every positive vector $\mathbf{b}$.

Lemma 3.3. Let $\mathcal{A} \in T(m, n)$ be an $M$-tensor and $\mathbf{b} \geq \mathbf{0}$. If there exists $\mathbf{v} \geq \mathbf{0}$ such that $\mathcal{A} \mathbf{v}^{m-1} \geq \mathbf{b}$, then the multilinear system of equations $\mathcal{A} \mathbf{x}^{m-1}=\mathbf{b}$ has a nonnegative solution.

By the above lemmas, we have the following theorems.

Theorem 3.4. Let $\mathcal{A} \in T(m, n)$. If $\mathcal{A}$ can be written as $\mathcal{A}=c \mathcal{I}-\mathcal{B}$ with $\mathcal{B} \geq 0$ and $c>\rho(\mathcal{B})+1$, then for every positive vector $\mathbf{b}$ the TAVE (1.2) has a unique positive solution.

Proof. Let $s=c-1$. Then $\mathcal{A}=c \mathcal{I}-\mathcal{B}$ yields

$$
\mathcal{A}-\mathcal{I}=s \mathcal{I}-\mathcal{B}, \quad \mathcal{B} \geq 0, \quad s>\rho(\mathcal{B}),
$$

which implies that $\mathcal{A}-\mathcal{I}$ is a strong $M$-tensor. By Lemma 3.1, the multilinear system of equations

$$
(\mathcal{A}-\mathcal{I}) \mathbf{x}^{m-1}=\mathbf{b}
$$

has a unique positive solution for every positive vector $\mathbf{b}$. Hence, for every positive vector $\mathbf{b}$, the TAVE (1.2) has a unique positive solution.

Combining [9, Theorem 3] and Lemma 3.2, we can rewrite the above theorem into an equivalent condition for $\mathcal{A}-\mathcal{I}$ being a strong $M$-tensor.

Theorem 3.5. Let $\mathcal{A} \in T(m, n)$ be a $Z$-tensor. Then $\mathcal{A}$ can be written as the form of

$$
\mathcal{A}=c \mathcal{I}-\mathcal{B}, \quad \mathcal{B} \geq 0, \quad c>\rho(\mathcal{B})+1
$$

if and only if for every positive vector $\mathbf{b}$ the TAVE (1.2) has a unique positive solution.

Proof. On one hand, by Theorem 3.4. we have the existence and uniqueness of the positive solution of the TAVE (1.2) for every positive vector $\mathbf{b}$. On the other hand, if 
for every positive vector $\mathbf{b}$ the TAVE (1.2) has a unique positive solution, then there exists a vector $\mathbf{x}>\mathbf{0}$ such that

$$
(\mathcal{A}-\mathcal{I}) \mathbf{x}^{m-1}=\mathbf{b}>\mathbf{0} .
$$

Since $\mathcal{A}$ is a $Z$-tensor, $\mathcal{A}-\mathcal{I}$ is also a $Z$-tensor. Thus, by [9, Theorem 3], $\mathcal{A}-\mathcal{I}$ is a strong $M$-tensor and then the form of (3.1) holds.

REMARK. The sufficient condition in Theorem 3.5 can be weakened as follows: if the TAVE (1.2) has a nonnegative solution for every positive vector $\mathbf{b}$, then we also have the form (3.1). In fact, let $\mathbf{x} \geq \mathbf{0}$ be a solution of the TAVE (1.2). Then there exists $\mathbf{x} \geq \mathbf{0}$ such that $(\mathcal{A}-\mathcal{I}) \mathbf{x}^{m-1}>\mathbf{0}$. By [9, Theorem 3], we can obtain the conclusion.

TheOREM 3.6. Let $\mathbf{b} \geq \mathbf{0}$ and $\mathcal{A} \in T(m, n)$ be in the form of $\mathcal{A}=c \mathcal{I}-\mathcal{B}$ with $\mathcal{B} \geq 0$ and $c=\rho(\mathcal{B})+1$. If there exists a vector $\mathbf{v} \geq \mathbf{0}$ such that $(\mathcal{A}-\mathcal{I}) \mathbf{v}^{m-1} \geq \mathbf{b}$, then the TAVE (1.2) has a nonnegative solution.

Proof. It follows from

$$
\mathcal{A}=c \mathcal{I}-\mathcal{B}, \quad \mathcal{B} \geq 0, \quad c=\rho(\mathcal{B})+1
$$

that $\mathcal{A}-\mathcal{I}$ is an $M$-tensor. By Lemma 3.3 , there is $\mathbf{x}^{*} \geq \mathbf{0}$ such that

$$
(\mathcal{A}-\mathcal{I})\left(\mathbf{x}^{*}\right)^{m-1}=\mathbf{b} .
$$

Thus, we have

$$
\mathcal{A}\left(\mathbf{x}^{*}\right)^{m-1}-\left|\mathbf{x}^{*}\right|^{m-1}=\mathbf{b} .
$$

This completes the proof.

We next extend the result (i) about the AVE (2.1) to the TAVE (1.2). Here, we assume that $m$ is even. We first introduce the product of a tensor and a diagonal tensor.

DeFINITION 3.7. Let $\mathcal{C}=\left(c_{i_{1} i_{2} \ldots i_{m}}\right) \in T(m, n)$ and $\mathcal{B} \in T(m, n)$ be a diagonal tensor with diagonal elements $b_{i \ldots i}$. We denote $\mathcal{C B}=\left(a_{i_{1} i_{2} \ldots i_{m}}\right)$ their product, whose elements are defined as

$$
a_{i_{1} i_{2} \ldots i_{m}}=c_{i_{1} i_{2} \ldots i_{m}}\left(b_{i_{2} \ldots i_{2}}\right)^{\frac{1}{m-1}} \cdots\left(b_{i_{m} \ldots i_{m}}\right)^{\frac{1}{m-1}}, \quad 1 \leq i_{1}, \cdots, i_{m} \leq n .
$$

Obviously, Definition 3.7 is well-defined due to the assumption that $m$ is even.

By simplicity computation, we have the following proposition.

Proposition 3.8. Let $\mathcal{C}=\left(c_{i_{1} i_{2} \ldots i_{m}}\right) \in T(m, n)$ and $\mathbf{x} \in R^{n}$. We have

$$
(\mathcal{C D}) \mathbf{x}^{m-1}=\mathcal{C}\left(\mathcal{D} \mathbf{x}^{m-1}\right)
$$

Proof. Let us define a vector $\mathbf{u} \in R^{n}$ as

$$
\mathbf{u}=\left(\mathcal{D} \mathbf{x}^{m-1}\right)^{\left[\frac{1}{m-1}\right]} .
$$

Then by some definitions introduced in Section 1, the $i$ th-component of the vector 
$\mathcal{C}\left(\mathcal{D} \mathbf{x}^{m-1}\right)$ can be written as

$$
\begin{aligned}
\left(\mathcal{C}\left(\mathcal{D} \mathbf{x}^{m-1}\right)\right)_{i} & =\left(\mathcal{C} \mathbf{u}^{m-1}\right)_{i} \\
& =\sum_{i_{2}=1}^{n} \cdots \sum_{i_{m}=1}^{n} c_{i i_{2} \ldots i_{m}} u_{i_{2}} \cdots u_{i_{m}} \\
& =\sum_{i_{2}=1}^{n} \cdots \sum_{i_{m}=1}^{n} c_{i i_{2} \ldots i_{m}}\left(d_{i_{2} \ldots i_{2}} x_{i_{2}}^{m-1}\right)^{\frac{1}{m-1}} \cdots\left(d_{i_{m} \ldots i_{m}} x_{i_{m}}^{m-1}\right)^{\frac{1}{m-1}} \\
& =\sum_{i_{2}=1}^{n} \cdots \sum_{i_{m}=1}^{n} c_{i i_{2} \ldots i_{m}} d_{i_{2} \ldots i_{2}}^{\frac{1}{m-1}} \cdots d_{i_{m} \ldots i_{m}}^{\frac{1}{m-1}} x_{i_{2}} \cdots x_{i_{m}} .
\end{aligned}
$$

Let $\mathcal{A}=\mathcal{C D}$. Then by Definition 3.7 the $i$ th-component of the vector $(\mathcal{C D}) \mathbf{x}^{m-1}$ can be written as

$$
\begin{aligned}
\left((\mathcal{C D}) \mathbf{x}^{m-1}\right)_{i} & =\sum_{i_{2}=1}^{n} \cdots \sum_{i_{m}=1}^{n} a_{i i_{2} \ldots i_{m}} x_{i_{2}} \cdots x_{i_{m}} \\
& =\sum_{i_{2}=1}^{n} \cdots \sum_{i_{m}=1}^{n} c_{i i_{2} \ldots i_{m}} d_{i_{2} \ldots i_{2}}^{\frac{1}{m-1}} \cdots d_{i_{m} \ldots i_{m}}^{\frac{1}{m-1}} x_{i_{2}} \cdots x_{i_{m}}
\end{aligned}
$$

Combining (3.2) and (3.3), we have

$$
(\mathcal{C D}) \mathbf{x}^{m-1}=\mathcal{C}\left(\mathcal{D} \mathbf{x}^{m-1}\right)
$$

口

It is easy to see that

$$
|\mathbf{x}|^{m-1}=\mathcal{D} \mathbf{x}^{m-1}
$$

holds for any vector $\mathbf{x} \in R^{n}$, because the $i$ th-component of the vectors $|\mathbf{x}|^{m-1}$ and $\mathcal{D} \mathbf{x}^{m-1}$ are in the form of

$$
\left(|\mathbf{x}|^{m-1}\right)_{i}=\left|x_{i}\right|^{m-1}, \quad\left(\mathcal{D} \mathbf{x}^{m-1}\right)_{i}=d_{i \ldots i} x_{i}^{m-1} .
$$

Here the sign of $x_{i}$ is corresponded to the diagonal element 1 or -1 of $\mathcal{D}$.

The following theorem is a generalization of the result (ii) from AVE to TAVE.

Theorem 3.9. Let $\mathcal{C} \in T(m, n), \mathbf{b} \in R^{n}$ and $\mathcal{A}=\mathcal{C D}$. If the multilinear system of equations

$$
(\mathcal{C}-\mathcal{I}) \mathbf{z}^{m-1}=\mathbf{b}, \quad \mathbf{z} \geq \mathbf{0}
$$

has a solution, then the tensor absolute value equation

$$
\mathcal{A} \mathbf{x}^{m-1}-|\mathbf{x}|^{m-1}=\mathbf{b}
$$

also has a solution.

Proof. Let $\mathbf{z}^{*}$ be the solution of the multilinear system of equations (3.5). Then we have

$$
(\mathcal{C}-\mathcal{I})\left(\mathbf{z}^{*}\right)^{m-1}=\mathbf{b}, \quad \mathbf{z}^{*} \geq \mathbf{0} .
$$

Take

$$
\mathcal{D}\left(\mathbf{x}^{*}\right)^{m-1}=\left(\mathbf{z}^{*}\right)^{m-1} .
$$


Then (3.6) can be rewritten as

$$
\mathcal{C}\left(\mathcal{D}\left(\mathbf{x}^{*}\right)^{m-1}\right)-\mathcal{D}\left(\mathbf{x}^{*}\right)^{m-1}=\mathbf{b},
$$

which, together with Proposition 3.8 and (3.4), implies that $\mathrm{x}^{*}$ is a solution of the tensor absolute value equation

$$
\mathcal{A} \mathbf{x}^{m-1}-|\mathbf{x}|^{m-1}=\mathbf{b}
$$

Thus, we complete the proof.

We give an example to verify the above theorem.

EXAMPLE 3.1. Let $\mathcal{C} \in T(4,2)$ with $c_{1111}=c_{1222}=c_{2111}=c_{2222}=1$ and zero otherwise, and $\mathbf{b}=(8,8)^{T}$. Consider the multilinear system of equations

$$
(\mathcal{C}-\mathcal{I}) \mathbf{z}^{m-1}=\mathbf{b}
$$

It is rewritten as

$$
z_{1}^{3}=8, \quad z_{2}^{3}=8
$$

This implies that $\mathbf{z}^{*}=(2,2)^{T}$ is a solution of

$$
(\mathcal{C}-\mathcal{I}) \mathbf{z}^{m-1}=\mathbf{b}, \quad \mathbf{z} \geq \mathbf{0} .
$$

Let $\mathcal{D} \in T(4,2)$ be a diagonal tensor with $d_{1111}=1$ and $d_{2222}=-1$. Then we have $\mathcal{A} \in T(4,2)$ with $a_{1111}=a_{2111}=1, a_{1222}=a_{2222}=-1$, and zero otherwise, i.e., $\mathcal{A}=\mathcal{C D}$. By Theorem [3.9, $\mathbf{x}^{*}=(2,-2)^{T}$ is just a solution of the tensor absolute value equation

$$
\mathcal{A} \mathbf{x}^{m-1}-|\mathbf{x}|^{m-1}=\mathbf{b} .
$$

We now verify the conclusion. We rewrite (3.7) as

$$
\left\{\begin{aligned}
x_{1}^{3}-x_{2}^{3}-\left|x_{1}\right|^{3} & =8 \\
-x_{2}^{3}+x_{1}^{3}-\left|x_{2}\right|^{3} & =8
\end{aligned}\right.
$$

By simplicity computation, the above equation has a solution $x_{1}=2, x_{2}=-2$.

4. Reformulation and algorithm. In this section, we extend the result (i) from AVE to TAVE. We show that the TAVE (1.2) is equivalent to a bi-multiliear program and a generalized tensor complementarity problem. We first introduce the following definition.

Definition 4.1. Let $\mathcal{A} \in T(m, n)$ and $\mathbf{x}, \mathbf{b} \in R^{n}$. Define

$$
F(\mathbf{x})=(\mathcal{A}+\mathcal{I}) \mathbf{x}^{m-1}-\mathbf{b}, \quad G(\mathbf{x})=(\mathcal{A}-\mathcal{I}) \mathbf{x}^{m-1}-\mathbf{b} .
$$

The generalized tensor complementarity problem is to find $\mathbf{x} \in R^{n}$ satisfying

$$
F(\mathbf{x}) \geq \mathbf{0}, \quad G(\mathbf{x}) \geq \mathbf{0}, \quad F(\mathbf{x})^{T} G(\mathbf{x})=0 .
$$

We call the following nonlinear program as a bi-multiliear program:

$$
0=\min \left\{F(\mathbf{x})^{T} G(\mathbf{x}) \mid F(\mathbf{x}) \geq \mathbf{0}, G(\mathbf{x}) \geq \mathbf{0}\right\}
$$


Theorem 4.2. Let $\mathcal{A} \in T(m, n)$ and $\mathbf{b} \in R^{n}$. Then the TAVE (1.2) is equivalent to the generalized tensor complementarity problem (4.1) and the bi-multilinear program (4.2).

Proof. Clearly, the generalized tensor complementarity problem (4.1) is equivalent to the bi-multilinear program (4.2). That is, (4.1) $\Leftrightarrow$ (4.2).

We only need to prove (1.2) $\Leftrightarrow(4.2)$. In fact, $|\mathbf{x}|^{m-1}=\left|\mathbf{x}^{m-1}\right|$. Hence, we have

$$
|\mathbf{x}|^{m-1} \leq \mathcal{A} \mathbf{x}^{m-1}-\mathbf{b} \Leftrightarrow(\mathcal{A}+\mathcal{I}) \mathbf{x}^{m-1}-\mathbf{b} \geq \mathbf{0}, \quad(\mathcal{A}-\mathcal{I}) \mathbf{x}^{m-1}-\mathbf{b} \geq \mathbf{0} .
$$

This implies that $\mathbf{x}$ is a feasible solution of (4.2). Since

$$
|\mathbf{x}|^{m-1}=\mathcal{A} \mathbf{x}^{m-1}-\mathbf{b} \Leftrightarrow\left((\mathcal{A}+\mathcal{I}) \mathbf{x}^{m-1}-\mathbf{b}\right)^{T}\left((\mathcal{A}-\mathcal{I}) \mathbf{x}^{m-1}-\mathbf{b}\right)=0,
$$

we have

$$
|\mathbf{x}|^{m-1}=\mathcal{A} \mathbf{x}^{m-1}-\mathbf{b} \Leftrightarrow 0=\min \left\{F(\mathbf{x})^{T} G(\mathbf{x}) \mid F(\mathbf{x}) \geq \mathbf{0}, G(\mathbf{x}) \geq \mathbf{0}\right\} .
$$

This completes the proof.

By the above theorem, in order to solve the TAVE (1.2), we propose an algorithm for solving the generalized tensor complementarity problem (4.1). Using the FischerBurmeister function $\phi_{F B}$, we can reformulate (4.1) as the following equation:

$$
H(\mathbf{x})=\left(\begin{array}{c}
\phi_{F B}\left(F_{1}(\mathbf{x}), G_{1}(\mathbf{x})\right) \\
\vdots \\
\phi_{F B}\left(F_{n}(\mathbf{x}), G_{n}(\mathbf{x})\right)
\end{array}\right)=\mathbf{0} .
$$

Hence, $\mathbf{x}$ is a solution of (1.2) if and only if $H(\mathbf{x})=\mathbf{0}$. Moreover, $H(\mathbf{x})$ is strongly semismooth since the composition of strongly semismooth function is again strongly semismooth [20, and according to the Jacobian chain rule, we have the following result.

Theorem 4.3. Let $\mathcal{A} \in S(m, n)$. Then the function $H(\mathbf{x})$ is strongly semismooth. Moreover, for any $\mathrm{x} \in R^{n}$, we have

$$
\partial H(\mathbf{x}) \subseteq D_{a}(\mathbf{x}) J F(\mathbf{x})+D_{b}(\mathbf{x}) J G(\mathbf{x}),
$$

where $D_{a}(\mathbf{x})=\operatorname{diag}\left(a_{i}(\mathbf{x})\right)$ and $D_{b}(\mathbf{x})=\operatorname{diag}\left(b_{i}(\mathbf{x})\right)$ are diagonal matrices in $R^{n \times n}$ with entries $\left(a_{i}(\mathbf{x}), b_{i}(\mathbf{x})\right) \in \partial \phi_{F B}\left(F_{i}(\mathbf{x}), G_{i}(\mathbf{x})\right)$, where $\partial \phi_{F B}\left(F_{i}(\mathbf{x}), G_{i}(\mathbf{x})\right)$ denotes the set $\partial \phi_{F B}(a, b)$ with $(a, b)$ being replaced by $\left(F_{i}(\mathbf{x}), G_{i}(\mathbf{x})\right)$, and $J F(\mathbf{x})$ and $J G(\mathbf{x})$ are given by

$$
J F(\mathbf{x})=(\mathcal{A}+\mathcal{I}) \mathbf{x}^{m-2}, \quad J G(\mathbf{x})=(\mathcal{A}-\mathcal{I}) \mathbf{x}^{m-2} .
$$

Here, for a tensor $\mathcal{T}=\left(t_{i_{1} \ldots i_{m}}\right) \in T(m, n)$ and a vector $\mathbf{x} \in R^{n}$, let $\mathcal{T} \mathbf{x}^{m-2}$ be a matrix in $R^{n \times n}$ whose $(i, j)$-th component is defined by

$$
\left(\mathcal{T} \mathbf{x}^{m-2}\right)_{i j}=\sum_{i_{3}}^{n} \cdots \sum_{i_{m}}^{n} t_{i j i_{3} \ldots i_{m}} x_{i_{3}} \cdots x_{i_{m}} .
$$

In order to propose an algorithm for the solution of $H(\mathbf{x})=\mathbf{0}$, we define a merit function as

$$
\Psi(\mathbf{x})=\frac{1}{2}\|H(\mathbf{x})\|^{2} .
$$


We present some properties of the merit function, which can be obtained by [․․, Theorem 2.2.4 and Theorem 2.6.6].

THEOREM 4.4. Let $\mathcal{A} \in S(m, n)$. Then the merit function $\Psi(\mathbf{x})$ is continuously differentiable with

$$
\nabla \Psi(\mathbf{x})=Q^{T} H(\mathbf{x})
$$

for any $Q \in \partial H(\mathbf{x})$.

We are now in the position to propose a Levenberg-Marquardt-type algorithm to solve the semismooth system of equations $H(\mathbf{x})=\mathbf{0}$, which is an extension of the nonsmooth inexact Levenberg-Marquardt-type method in [11. To ensure global convergence, a line search is performed to minimize the smooth merit function $\Psi$. Because the problem with data in a structure of tensor is large scale, and the inexact version is more suited to the large-scale case [11, we have the following algorithm.

Algorithm 4.1. (Inexact Levenberg-Marquardt-type method)

Step 0. Given a starting vector $\mathbf{x}^{0} \in R^{n}$ and some scales $p>2,0<\beta<1 / 2, \rho>0$, $\epsilon \geq 0$. Set $k:=0$.

Step 1. If $\left\|H\left(\mathbf{x}^{k}\right)\right\| \leq \epsilon$, stop. Otherwise, compute $Q^{k} \in \partial H\left(\mathbf{x}^{k}\right)$.

Step 2. Find a solution $\mathbf{d}^{k}$ satisfying

$$
\left(\left(Q^{k}\right)^{T} Q^{k}+\mu_{k} I\right) \mathbf{d}=-\left(Q^{k}\right)^{T} H\left(\mathbf{x}^{k}\right)+\mathbf{r}^{k},
$$

where $\mu_{k} \geq 0$ is the Levenberg-Marquardt parameter. If the condition

$$
\nabla \Psi\left(\mathbf{x}^{k}\right)^{T} \mathbf{d}^{k} \leq-\rho\left\|\mathbf{d}^{k}\right\|^{p}
$$

is not satisfied, set

$$
\mathbf{d}^{k}=-\nabla \Psi\left(\mathbf{x}^{k}\right) .
$$

Step 3. Find the smallest integer $i^{k} \in\{0,1,2, \ldots\}$ such that $t_{k}=2^{-i^{k}}$ and

$$
\Psi\left(\mathbf{x}^{k}+t_{k} \mathbf{d}^{k}\right) \leq \Psi\left(\mathbf{x}^{k}\right)+\beta t_{k} \nabla \Psi\left(\mathbf{x}^{k}\right)^{T} \mathbf{d}^{k} .
$$

Step 4. Set $\mathbf{x}^{k+1}=\mathbf{x}^{k}+t_{k} \mathbf{d}^{k}, k:=k+1$, and go to Step 1 .

In what follows, we analyze the global convergence of Algorithm 1. We shall assume that Algorithm 1 produce an infinite sequence $\left\{\mathbf{x}^{k}\right\}$. By [11, Theorem 15 and Theorem 16], we immediately obtain the following theorems.

THEOREM 4.5. Assume that the sequence $\left\{\mu_{k}\right\}$ is bounded and that the sequence $\left\{\mathbf{r}^{k}\right\}$ satisfies

$$
\left\|\mathbf{r}^{k}\right\| \leq \alpha_{k}\left\|\nabla \Psi\left(\mathbf{x}^{k}\right)\right\|
$$

where $\left\{\alpha_{k}\right\}$ is a sequence of numbers with $0<\alpha_{k}<1$ and $\alpha_{k} \rightarrow 0$ as $k \rightarrow \infty$. Then each accumulation point of $\left\{\mathbf{x}^{k}\right\}$ is a stationary point of $\Psi$.

THEOREM 4.6. Let the assumptions of Theorem 4.5 hold. If one of the accumulation points of $\left\{\mathbf{x}^{k}\right\}$, denoted $\mathbf{x}^{*}$, is an isolated solution of the TAVE (1.2), then

$$
\lim _{k \rightarrow \infty} \mathbf{x}^{k}=\mathbf{x}^{*}
$$

In the implementation of Algorithm 4.1, the computational most intensive part is the approximation solution of system (4.3) with $\mathbf{r}^{k}=\mathbf{0} \quad \forall k$. We note that the system 
is always solvable. In fact, if $\mu_{k}>0$, the matrix $\left(Q^{k}\right)^{T} Q^{k}+\mu_{k} I$ is symmetric positive definite and hence system (4.3) is surely solvable. If $\mu_{k}=0$, the matrix $\left(Q^{k}\right)^{T} Q^{k}+\mu_{k} I$ reduces to $\left(Q^{k}\right)^{T} Q^{k}$, which is guaranteed to be only positive semidefinite. However, in this case, (4.3) reduces to the normal gradient equation $Q^{k} \mathbf{d}=-H\left(\mathbf{x}^{k}\right)$, is therefore solvable. We now have to specify which element $Q^{k} \in \partial H\left(\mathbf{x}^{k}\right)$ we select at the $k$-th iteration. By Theorem 4.3. we have that an element of $\partial H\left(\mathbf{x}^{k}\right)$ can be obtained in the following way. Let

$$
\Lambda=\left\{i: F_{i}\left(\mathbf{x}^{k}\right)=0=G_{i}\left(\mathbf{x}^{k}\right)\right\}
$$

be the set of "degenerate indices" and define $\mathbf{z} \in R^{n}$ to be a vector whose components $z_{i}$ are 1 if $i \in \Lambda$ and 0 otherwise. Then, the matrix $Q^{k}$ defined by

$$
Q^{k}=A\left(\mathbf{x}^{k}\right) J F\left(\mathbf{x}^{k}\right)+B\left(\mathbf{x}^{k}\right) J G\left(\mathbf{x}^{k}\right),
$$

where $A$ and $B$ are $n \times n$ diagonal matrices whose $i$-th diagonal elements are given, respectively, by

$$
A_{i i}\left(\mathbf{x}^{k}\right)= \begin{cases}1-\frac{F_{i}\left(\mathbf{x}^{k}\right)}{\sqrt{F_{i}^{2}\left(\mathbf{x}^{k}\right)+G_{i}^{2}\left(\mathbf{x}^{k}\right)}} & \text { if } i \notin \Lambda, \\ 1-\frac{\nabla F_{i}\left(\mathbf{x}^{k}\right)^{T} z}{\sqrt{\left(\nabla F_{i}\left(\mathbf{x}^{k}\right)^{T} z\right)^{2}+\left(\nabla G_{i}\left(\mathbf{x}^{k}\right)^{T} z\right)^{2}}} & \text { if } i \in \Lambda,\end{cases}
$$

and by

$$
B_{i i}\left(\mathbf{x}^{k}\right)= \begin{cases}1-\frac{G_{i}\left(\mathbf{x}^{k}\right)}{\sqrt{F_{i}^{2}\left(\mathbf{x}^{k}\right)+G_{i}^{2}\left(\mathbf{x}^{k}\right)}} & \text { if } i \notin \Lambda, \\ 1-\frac{\nabla G_{i}\left(\mathbf{x}^{k}\right)^{T} z}{\sqrt{\left(\nabla F_{i}\left(\mathbf{x}^{k}\right)^{T} z\right)^{2}+\left(\nabla G_{i}\left(\mathbf{x}^{k}\right)^{T} z\right)^{2}}} & \text { if } i \in \Lambda,\end{cases}
$$

belongs to $\partial H\left(\mathbf{x}^{k}\right)$. In the next section, we compute $Q^{k}$ as the formulation.

5. Numerical results. In this section, we present the numerical performance of Algorithm 4.1 for the TAVE (1.2). All codes were written by using Matlab Version R2015b and Tensor Toolbox Version 2.6 [1. The numerical experiments were done on a laptop with an Intel Core i7-4720HQ CPU $(2.6 \mathrm{GHz})$ and RAM of 7.89GB.

In the implementation of Algorithm 4.1, we set $\varepsilon=10^{-6}, \rho=10^{-10}, p=2.1, \beta=$ $10^{-4}$ and the Levenberg-Marquardt parameter $\mu_{k}=0.3 \forall k \in N$. We also set a maximum iteration steps for the algorithm, i.e., $N_{\max }=300$.

The first numerical experiment focuses on the behaviour of algorithm's iteration. We generate a random symmetric nonnegative tensor $\mathcal{A} \in S_{6,8}$ and a random vector $\mathrm{x}^{*} \in R^{8}$. All entries of $\mathcal{A}$ and $\mathrm{x}^{*}$ are uniform random numbers in the interval [0,1]. We calculate $\mathbf{b}=\mathcal{A} \mathbf{x}^{* m-1}-\left|\mathbf{x}^{*}\right|^{m-1}$ in order to make TAVE have at least one solution. Then we use Algorithm 4.1 to solve TAVE: $\mathcal{A} \mathbf{x}^{m-1}-|\mathbf{x}|^{m-1}=\mathbf{b}$, with a random initial point chosen randomly from $[0,1]^{8}$ which is shown as $x^{0}$ in table [5.1. The iteration of Algorithm 4.1 is shown in Table 5.1 From the table, $\left\|H\left(\mathbf{x}^{k}\right)\right\|$ tends to 0 as the number of iteration $k$ increases. And $\left\|\nabla \Psi\left(\mathbf{x}^{k}\right)\right\|$ also tends to 0 except that it increases from $\mathbf{k}=2$ to $\mathbf{k}=4$. This shows that $\left\|\nabla \Psi\left(\mathbf{x}^{k}\right)\right\|$ does converge to 0 but not converge monotonically when the algorithm converges.

The second numerical experiment aims to verify Theorem 3.9. We first generate a random symmetric nonnegative tensor $\mathcal{C} \in S(4,10)$ and a random vector $\mathbf{z}^{*}=$ $(0.1040,0.7455,0.7363,0.5619,0.1842,0.5972,0.2999,0.1341,0.2126,0.8949)^{\mathrm{T}} \in R^{10}$. All entries of $\mathcal{C}$ are uniform random numbers in the interval $[0,1]$. Let $\mathbf{b}=(\mathcal{C}-$ 
TABLE 5.1

Iterations of Algorithm 4.1 for a random tensor $\mathcal{A} \in S_{6,8}$ and corresponding $\mathbf{b}$

\begin{tabular}{cccc}
\hline $\mathbf{k}$ & $\mathbf{x}^{k}$ & $\left\|H\left(\mathbf{x}^{k}\right)\right\|$ & $\left\|\nabla \Psi\left(\mathbf{x}^{k}\right)\right\|$ \\
\hline 0 & $(0.8143,0.2435,0.9293,0.3500,0.1966,0.2511,0.6160,0.4733)^{\mathrm{T}}$ & 562.2589 & 1500602.8826 \\
1 & $(0.7407,0.2435,0.6880,0.3545,0.1072,0.3670,0.4555,0.3271)^{\mathrm{T}}$ & 148.1702 & 203193.6101 \\
2 & $(0.4542,0.4477,0.4349,0.4348-0.2944,0.7819,0.4209,0.3007)^{\mathrm{T}}$ & 25.5263 & 23486.6536 \\
3 & $(1.0757,0.3147,0.2655,0.4343-0.2368,0.4908,0.1690,0.3781)^{\mathrm{T}}$ & 20.2932 & 48494.4079 \\
4 & $(1.2158,0.3379,0.4481,0.5825-0.2075,0.1750,0.3290,0.0197)^{\mathrm{T}}$ & 18.6526 & 49990.1630 \\
5 & $(0.8865,0.3812,0.3176,0.5179-0.3075,0.3684,0.4486,0.2840)^{\mathrm{T}}$ & 10.0354 & 20912.3905 \\
6 & $(0.8742,0.2928,0.3744,0.5308-0.3895,0.5269,0.2838,0.3997)^{\mathrm{T}}$ & 2.9292 & 3867.9206 \\
7 & $(0.8798,0.2888,0.3406,0.6301-0.3722,0.4799,0.3198,0.3293)^{\mathrm{T}}$ & 1.3213 & 1522.0099 \\
8 & $(0.8664,0.2829,0.3003,0.6746-0.3890,0.4936,0.3325,0.3355)^{\mathrm{T}}$ & 0.7455 & 1084.3075 \\
9 & $(0.8684,0.2850,0.2737,0.6914-0.3985,0.4960,0.3394,0.3411)^{\mathrm{T}}$ & 0.1766 & 482.0095 \\
10 & $(0.8690,0.2852,0.2752,0.6895-0.3976,0.4957,0.3383,0.3411)^{\mathrm{T}}$ & 0.0144 & 21.2907 \\
11 & $(0.8692,0.2853,0.2753,0.6894-0.3975,0.4956,0.3383,0.3410)^{\mathrm{T}}$ & 0.0029 & 2.4370 \\
12 & $(0.8692,0.2853,0.2754,0.6893-0.3975,0.4956,0.3383,0.3410)^{\mathrm{T}}$ & 0.0002 & 0.1396 \\
13 & $(0.8692,0.2853,0.2754,0.6892-0.3975,0.4956,0.3383,0.3410)^{\mathrm{T}}$ & 0.0001 & 0.0008 \\
14 & $(0.8692,0.2853,0.2754,0.6892-0.3975,0.4956,0.3383,0.3410)^{\mathrm{T}}$ & 0.0000 & 0.0000 \\
\hline
\end{tabular}

$\mathcal{I}) \mathbf{z}^{* m-1}$. Since $\mathcal{D} \in S(4,10)$ is a diagonal tensor whose diagonal elements are 1 or -1 , there are at most $2^{10}=1024$ different $\mathcal{D}$. The first attempt is to generate all these 1024 tensors. For each tensor $\mathcal{D}_{k}$, set $\mathcal{A}_{k}=\mathcal{C D}_{k}$ (see Definition 3.7) and $\mathbf{x}_{\mathbf{k}}=\left(\mathcal{D}_{k} \mathbf{z}^{* m-1}\right)^{\frac{1}{m-1}}$. We check whether $\mathcal{A}_{k} \mathbf{x}_{\mathbf{k}}{ }^{m-1}-\left|\mathbf{x}_{\mathbf{k}}\right|^{m-1}$ is equals to $\mathbf{b}$ for all $k \in\{1,2, \ldots, 1024\}$. The result shows that each $\mathbf{x}_{\mathbf{k}}$ is just one of the solution to the corresponding TAVE problem $\mathcal{A}_{k} \mathbf{x}^{m-1}-|\mathbf{x}|^{m-1}=\mathbf{b}_{k}$.

The second attempt of the second numerical experiment is to generate five $\mathcal{D}_{k}$ of all 1024 tensors randomly and use Algortihm 4.1 to solve the corresponding TAVE. The diagonal elements of the five $\mathcal{D}_{k}$ is shown in Table 5.2 .

TABLE 5.2

Diagonal elements of $\mathcal{D}_{k}$

\begin{tabular}{cc}
\hline $\mathbf{k}$ & diag of $\mathcal{D}_{k}$ \\
\hline 1 & $(-1,-1,-1,-1,-1,-1,-1,-1,-1,-1)^{\mathrm{T}}$ \\
2 & $(-1,1,-1,1,-1,1,-1,-1,-1,1)^{\mathrm{T}}$ \\
3 & $(1,1,-1,-1,1,1,-1,-1,-1,-1)^{\mathrm{T}}$ \\
4 & $(-1,1,-1,1,-1,1,-1,-1,1,1)^{\mathrm{T}}$ \\
5 & $(1,-1,1,1,1,1,-1,1,-1,1)^{\mathrm{T}}$ \\
\hline
\end{tabular}

We first select the initial points for Algorithm 4.1 by using normal distribution, i.e., entries are from standardized normal distribution $N(0,1)$ independently. Here we call these initial points type-I initial points. The results of corresponding TAVE with type- $I$ initial points is summarized in Table 5.3. We can easily find out that none of the five $\mathbf{x}_{\mathbf{k}}$ is in the form of $\left(\mathcal{D}_{k} \mathbf{z}^{* m-1}\right)^{\frac{1}{m-1}}$. Because Algorithm 4.1 is based on the thoughts of Newton method, thus its convergence relies heavily on the initial point. In order to detect solution which is mentioned in Theorem 3.9 by Algorithm 4.1. we should choose the initial points in another way. For each $\mathcal{D}_{k}$, we generate type$I I$ initial points by adding a random number chosen from uniform distribution over $(-0.3,0.3)$ to $\left(\mathcal{D}_{k} \mathbf{z}^{* m-1}\right)^{\frac{1}{m-1}}$. The results of corresponding TAVE with type-II initial 
points is shown in Table 5.4. The solutions are exactly in the form of $\left(\mathcal{D}_{k} \mathbf{z}^{* m-1}\right)^{\frac{1}{m-1}}$.

TABLE 5.3

Numerical results for tensors $\mathcal{A}_{k}$ with type-I initial points

\begin{tabular}{|c|c|c|c|c|}
\hline $\mathrm{k}$ & $\mathrm{x}_{\mathrm{k}}$ & $\left\|H\left(\mathbf{x}_{\mathbf{k}}\right)\right\|$ & Iter. & Time \\
\hline 1 & $\begin{array}{l}(-0.3485,-0.0971,-0.7753,-1.2447,-0.7739 \\
-0.5628,-0.4868,0.4480,0.2925,-0.9003)^{\mathrm{T}}\end{array}$ & 0.00000012 & 15 & 0.2135 \\
\hline 2 & $\begin{array}{l}(0.4184,-0.0423,-0.2989,1.0357,-1.0340 \\
0.3109,-0.3686,-0.2755,-0.6852,0.9528)^{\mathrm{T}}\end{array}$ & 0.00000022 & 15 & 0.2060 \\
\hline 3 & $\begin{array}{c}(0.7454,0.5055,-0.6641,0.3093,-0.1769 \\
1.1273,-0.4514,-1.1430,-0.0619,-0.2421)^{\mathrm{T}}\end{array}$ & 0.00000003 & 18 & 0.2673 \\
\hline 4 & $\begin{array}{l}(-0.9570,0.5494,-2.1429,-0.1959,-1.8247 \\
-0.3996,0.8803,-0.3457,0.0458,0.1694)^{\mathrm{T}}\end{array}$ & 0.00000000 & 11 & 0.1355 \\
\hline 5 & $\begin{array}{c}(0.3385,-1.1498,1.0413,0.3533,0.7606, \\
-0.1214,-0.3290,-0.0458,-0.2049,0.4027)^{\mathrm{T}}\end{array}$ & 0.00000006 & 10 & 0.1265 \\
\hline
\end{tabular}

TABLE 5.4

Numerical results for tensors $\mathcal{A}_{k}$ with type-II initial points

\begin{tabular}{ccccc}
\hline $\mathbf{k}$ & $\mathbf{x}_{\mathbf{k}}$ & $\left\|H\left(\mathbf{x}_{\mathbf{k}}\right)\right\|$ & Iter. & Time \\
\hline \multirow{2}{*}{1} & $(-0.1040,-0.7455,-0.7363,-0.5619,-0.1842$, & & \\
& $-0.5972,-0.2999,-0.1341,-0.2126,-0.8949)^{\mathrm{T}}$ & 0.00000072 & 20 & 0.2523 \\
2 & $(-0.1040,0.7455,-0.7363,0.5619,-0.1842$, & & & \\
& $0.5972,-0.2999,-0.1341,-0.2126,0.8949)^{\mathrm{T}}$ & 0.00000090 & 17 & 0.2050 \\
3 & $(0.1040,0.7455,-0.7363,-0.5619,0.1842$, & & & \\
& $0.5972,-0.2999,-0.1341,-0.2126,-0.8949)^{\mathrm{T}}$ & 0.00000091 & 24 & 0.2838 \\
4 & $(-0.1040,0.7455,-0.7363,0.5619,-0.1842$, \\
& $0.5972,-0.2999,-0.1341,0.2126,0.8949)^{\mathrm{T}}$ & 0.00000064 & 16 & 0.1896 \\
5 & $(0.1040,-0.7455,0.7363,0.5619,0.1842$, & & & \\
& $0.5972,-0.2999,0.1341,-0.2126,0.8949)^{\mathrm{T}}$ & 0.00000075 & 14 & 0.1638 \\
\hline
\end{tabular}

In Tables 5.3 and 5.4, $\mathbf{k}$ denotes the experiment No. corresponding to Table 5.2 $\mathbf{x}_{\mathbf{k}}$ denotes the solution vectors returned by Algorithm 4.1. $\left\|H\left(\mathbf{x}_{\mathbf{k}}\right)\right\|$ denotes the Euclid norm of $H\left(\mathbf{x}_{\mathbf{k}}\right)$. If the norm of $H\left(\mathbf{x}_{\mathbf{k}}\right)$ is small enough, we can regard $\mathbf{x}_{\mathbf{k}}$ as an approximate solution of TAVE. Iter. denotes the number of iteration and Time denotes the time of iteration that finds corresponding $x$ by Algorithm 4.1. In the second experiment, we verify Theorem 3.9 from the instant correctly. Besides, from Table 5.3 and 5.4 we find that under the conditions of Theorem 3.9. the solution $\left(\mathcal{D} \mathbf{z}^{* m-1}\right)^{\frac{1}{m-1}}$ may not be the only solution of TAVE $\mathcal{A} \mathbf{x}^{m-1}-|\mathbf{x}|^{m-1}=\mathbf{b}$. There might be some other solutions, such as the solution in Table 5.3. To discuss the uniqueness of the positive solution, we conduct our third experiment.

Our third numerical experiment focuses on Theorem 3.5. Here we first generate a random symmetric nonnegative tensor $\mathcal{B}$ whose entries are uniform random numbers in the interval $[0,1]$. Let $c=1+(1+0.01) \max _{1 \leq i \leq n}\left(\mathcal{B} e^{3}\right)_{i}$, where $e=(1,1,1,1)^{\mathrm{T}}$. Since $\max _{1 \leq i \leq n}\left(\mathcal{B} e^{3}\right)_{i} \geq \rho(\mathcal{B})$, the choice of $c$ makes sure that $c>\rho(\mathcal{B})+1$. Then let $\mathcal{A}=c \mathcal{I}-\mathcal{B}$, and $\mathcal{A}$ satisfies the conditions of Theorem 3.5. i.e., $\mathcal{A}-\mathcal{I}$ is strong M-tensor. Tensors $\mathcal{B}$ and $\mathcal{A}$ are given in Tables 5.5 and 5.6. respectively. 
TABLE 5.5

$A$ random symmetric nonnegative tensor $\mathcal{B}=\left(b_{i_{1} i_{2} i_{3} i_{4}}\right) \in S(4,4)$

\begin{tabular}{lllll}
\hline$b_{1111}=0.8147$ & $b_{1112}=0.9058$ & $b_{1113}=0.1270$ & $b_{1114}=0.9134$ & $b_{1122}=0.6324$ \\
$b_{1123}=0.0975$ & $b_{1124}=0.2785$ & $b_{1133}=0.5469$ & $b_{1134}=0.9575$ & $b_{1144}=0.9649$ \\
$b_{1222}=0.1576$ & $b_{1223}=0.9706$ & $b_{1224}=0.9572$ & $b_{1233}=0.4854$ & $b_{1234}=0.8003$ \\
$b_{1244}=0.1419$ & $b_{1333}=0.4218$ & $b_{1334}=0.9157$ & $b_{1344}=0.7922$ & $b_{1444}=0.9595$ \\
$b_{2222}=0.6557$ & $b_{2223}=0.0357$ & $b_{2224}=0.8491$ & $b_{2233}=0.9340$ & $b_{2234}=0.6787$ \\
$b_{2244}=0.7577$ & $b_{2333}=0.7431$ & $b_{2334}=0.3922$ & $b_{2344}=0.6555$ & $b_{2444}=0.1712$ \\
$b_{3333}=0.7060$ & $b_{3334}=0.0318$ & $b_{3344}=0.2769$ & $b_{3444}=0.0462$ & $b_{4444}=0.0971$ \\
\hline \multicolumn{5}{c}{ TABLE 5.6 } \\
\\
The symmetric tensor $\mathcal{A}=\left(a_{i_{1} i_{2} i_{3} i_{4}}\right) \in S(4,4)$ based on B \\
\hline$a_{1111}=40.8037$ & $a_{1112}=-0.9058$ & $a_{1113}=-0.1270$ & $a_{1114}=-0.9134$ & $a_{1122}=-0.6324$ \\
$a_{1123}=-0.0975$ & $a_{1124}=-0.2785$ & $a_{1133}=-0.5469$ & $a_{1134}=-0.9575$ & $a_{1144}=-0.9649$ \\
$a_{1222}=-0.1576$ & $a_{1223}=-0.9706$ & $a_{1224}=-0.9572$ & $a_{1233}=-0.4854$ & $a_{1234}=-0.8003$ \\
$a_{1244}=-0.1419$ & $a_{1333}=-0.4218$ & $a_{1334}=-0.9157$ & $a_{1344}=-0.7922$ & $a_{1444}=-0.9595$ \\
$a_{2222}=40.9627$ & $a_{2223}=-0.0357$ & $a_{2224}=-0.8491$ & $a_{2233}=-0.9340$ & $a_{2234}=-0.6787$ \\
$a_{2244}=-0.7577$ & $a_{2333}=-0.7431$ & $a_{2334}=-0.3922$ & $a_{2344}=-0.6555$ & $a_{2444}=-0.1712$ \\
$a_{3333}=40.9124$ & $a_{3334}=-0.0318$ & $a_{3344}=-0.2769$ & $a_{3444}=-0.0462$ & $a_{4444}=41.5213$ \\
\hline
\end{tabular}

We choose 10 random positive vectors $\mathbf{b}_{k} \in R_{+}^{4}, k=1, \ldots, 10$. For each $\mathbf{b}_{k}$, we find 20 repeatable solutions of TAVE: $\mathcal{A} \mathbf{x}^{m-1}-|\mathbf{x}|^{m-1}=\mathbf{b}_{k}$ with random vectors from $N(0,1)^{4}$ as initial points repeatedly and summarize the results in Table 5.7

TABLE 5.7

Numerical results for the third experiment

\begin{tabular}{cccccc}
\hline $\mathbf{x}$ & $\mathbf{b}$ & Iter. & Time & $\max \|H(\mathbf{x})\|$ & Attempts \\
\hline$(0.8100,0.7881,0.7786,0.8003)^{\mathrm{T}}$ & $(1.4193,0.2916,0.1978,1.5877)^{\mathrm{T}}$ & 31.00 & 0.6783 & 0.00000098 & $20 / 100$ \\
$(0.7285,0.7212,0.7156,0.7098)^{\mathrm{T}}$ & $(0.8045,0.6966,0.8351,0.2437)^{\mathrm{T}}$ & 19.40 & 0.3109 & 0.00000099 & $20 / 157$ \\
$(0.7219,0.7313,0.7230,0.7098)^{\mathrm{T}}$ & $(0.2157,1.1658,1.1480,0.1049)^{\mathrm{T}}$ & 19.55 & 0.3456 & 0.00000099 & $20 / 205$ \\
$(0.8453,0.8603,0.8294,0.8276)^{\mathrm{T}}$ & $(0.7223,2.5855,0.6669,0.1873)^{\mathrm{T}}$ & 13.65 & 0.1907 & 0.00000082 & $20 / 244$ \\
$(0.8445,0.8584,0.8321,0.8507)^{\mathrm{T}}$ & $(0.0825,1.9330,0.4390,1.7947)^{\mathrm{T}}$ & 14.05 & 0.2168 & 0.00000084 & $20 / 290$ \\
$(0.7104,0.7055,0.6849,0.6957)^{\mathrm{T}}$ & $(0.8404,0.8880,0.1001,0.5445)^{\mathrm{T}}$ & 68.25 & 1.7492 & 0.00000051 & $20 / 145$ \\
$(0.6775,0.6771,0.6677,0.6750)^{\mathrm{T}}$ & $(0.3035,0.6003,0.4900,0.7394)^{\mathrm{T}}$ & 21.75 & 0.3864 & 0.00000099 & $20 / 216$ \\
$(0.9021,0.8787,0.8894,0.8805)^{\mathrm{T}}$ & $(1.7119,0.1941,2.1384,0.8396)^{\mathrm{T}}$ & 15.70 & 0.2535 & 0.00000089 & $20 / 114$ \\
$(0.8104,0.8007,0.7908,0.7841)^{\mathrm{T}}$ & $(1.3546,1.0722,0.9610,0.1240)^{\mathrm{T}}$ & 14.60 & 0.2121 & 0.00000071 & $20 / 129$ \\
$(0.8957,0.8939,0.8661,0.8808)^{\mathrm{T}}$ & $(1.4367,1.9609,0.1977,1.2078)^{\mathrm{T}}$ & 13.60 & 0.1980 & 0.00000099 & $20 / 114$ \\
\hline
\end{tabular}

In Table 5.7 $\mathbf{x}$ denotes the solution of TAVE. $\mathbf{b}$ denotes random generated $\mathbf{b}_{k}$ mentioned above. Iter. denotes the average number of iteration that finds the corresponding solution successfully. Time denotes the average time of iteration that finds the corresponding solution by Algorithm 1. $\max \|H(\mathbf{x})\|$ is the maximum norm of all $H(\mathbf{x})$ returned by Algorithm 1 whose $\mathbf{x}$ is the corresponding solution. Attempts has the form $\mathbf{N} / \mathbf{T}, \mathbf{N}$ denotes the number of the corresponding $\mathbf{x}$ found by Algorithm 4.1 and $\mathbf{T}$ denotes the number of initial points in all.

In this experiment, we use "while" loop in Matlab program to guarantee that we can get exact 20 solutions (might be repeatable) for each $\mathbf{b}_{\mathbf{k}} \geq \mathbf{0}$. According to Table 5.7. for each $\mathbf{b}_{\mathbf{k}} \geq \mathbf{0}$, Algorithm 4.1 only returns unique positive solution in all 20 repeatable solutions. This phenomenon fits Theorem 3.5 very well. Besides, in order 
to get 20 valid solutions, the initial points we attempt is 10 times more than the valid ones. This means that most of the random initial points fail to find a solution by Algorithm 4.1. The reason might be that the convergence of Newton type method depends on the initial point badly. Theorem 3.5 shows that under this circumstances, there's only one unique positive solution of TAVE. If and only if the initial point is in the convergence region of some solution of TAVE, the algorithm will converge. Therefore, it's harder to find valid solutions if $\mathbf{b} \geq \mathbf{0}$. Table 5.8 shows the solutions found by Algorithm 4.1 when $\mathbf{b}=(-1,1,1,1)$. The initial points attempted in all is much less.

TABLE 5.8

Solutions of TAVE when $\mathbf{b}=(-1,1,1,1)$

\begin{tabular}{cccccc}
\hline $\mathbf{x}$ & $\mathbf{b}$ & Iter. & Time & $\max \|H(\mathbf{x})\|$ & Attempts \\
\hline$(0.0800,0.3629,0.3543,0.3505)^{\mathrm{T}}$ & $(-1,1,1,1)^{\mathrm{T}}$ & 12.67 & 0.1644 & 0.00000070 & $3 / 20$ \\
$(-0.2593,0.2948,0.2891,0.2903)^{\mathrm{T}}$ & $(-1,1,1,1)^{\mathrm{T}}$ & 13.67 & 0.1708 & 0.00000099 & $3 / 20$ \\
$(0.6258,0.6600,0.6522,0.6537)^{\mathrm{T}}$ & $(-1,1,1,1)^{\mathrm{T}}$ & 11.93 & 0.1516 & 0.00000075 & $14 / 20$ \\
\hline
\end{tabular}

Moreover, under the circumstances that $\mathbf{b} \geq \mathbf{0}$ and $\mathcal{A}-\mathcal{I}$ is strong M-tensor, whether the unique positive solution of TAVE is the unique solution of TAVE remains a question. In our experiment we haven't found other solutions except for the unique positive ones.

6. Conclusion. We have introduced tensor absolute value equations. The simple definition is a natural generalization of the definition of absolute value equations in the matrix case. We have established some basic properties for tensor absolute value equations and we reformulate tensor absolute value equations as a generalized tensor complementarity problem. We have proposed some sufficient conditions for the existence of solution to the multilinear equations. We propose an inexact LevenbergMarquardt-type method (Algorithm 4.1) to solve the tensor absolute value equations and some numerical results have shown that our algorithm is performing well.

There are some questions which are still in study. For example, we known that "The AVE (2.1) is uniquely solvable for any $\mathbf{b} \in R^{n}$ if the singular values of $A$ exceed 1" 19. Can we extend the conclusion to TVAE (1.2), i.e., the statement "The TAVE (1.2) is uniquely solvable for any $\mathbf{b} \in R^{n}$ if the singular values of tensor $\mathcal{A}$ exceed 1 " is correct or not? This is still an open question.

Acknowledgments. Shouqiang Du's work was supported by the National Natural Science Foundation of China (Grant No. 11671220, 11401331) and the Nature Science Foundation of Shandong Province (ZR2015AQ013, ZR2016AM29). Liping Zhang's work was supported by the National Natural Science Foundation of China (Grant No. 11271221). Liqun Qi's work was supported by the Hong Kong Research Grant Council (Grant No. PolyU 501212, 501913, 15302114 and 15300715).

\section{REFERENCES}

[1] B.W. BAder, T.G. Kolda, et al., MATLAB Tensor Toolbox Version 2.6 (2012). http://www.sandia.gov/ tgkolda/TensorToolbox/

[2] J.F. Bonnans, R. Cominetti, And A. Shapiro, Second order optimality conditions based on parabolic second order tangent sets, SIAM Journal on Optimization, 9 (1999), pp. 466-493.

[3] K. Chang, L. QI, And T. Zhang, A survey on the spectral theory of nonnegative tensors, Numerical Linear Algebra with Applications, 20 (2013), pp. 891-912. 
[4] M. Che, L. QI, And Y. WeI, Positive definite tensors to nonlinear complementarity problems, Journal of Optimization Theory and Applications, 168 (2016), pp. 475-487.

[5] M. Che And L. QI, A semismooth Newton method for tensor eigenvalue complementarity problem, Computational Optimization and Applications, 65 (2016), pp. 109-126.

[6] F.H. Clarke, Optimization and Nonsmooth Analysis, Wiley, New York, 1983.

[7] R.W. Cottle, J.-S. PAng, and R.E. Stone, The Linear Complementarity Problem, Academic Press, Boston, 1992.

[8] W. Ding And Y. WeI, Solving multi-linear systems with $M$-Tensors, Journal of Scientific Computing, 68 (2016), pp. 683-715.

[9] W. Ding, L. QI, AND Y. WeI, M-tensors and nonsingular M-tensors, Linear Algebra and Its Applications, 439 (2013), pp. 3264-3278.

[10] F. FACChinei AND J.-S. PANG, Finite-Dimensional Variational Inequalities and Complementarity Problems, Springer-Verlag, New York, 2003.

[11] F. FaCChinei And C. Kanzow, A nonsmooth inexact Newton method for the solution of largescale nonlinear complementarity problems, Mathematical Programming, 76 (1997), pp. 493512.

[12] A. Fischer, A special Newton-type optimization method, Optimization, 24 (1992), pp. 269-284.

[13] M. Hintermüller, Semismooth Newton methods and applications, Department of Mathematics, Humboldt-University of Berlin, 2010.

[14] Z. HUANG AND L. QI, Formulating an n-person noncooperative game as a tensor complementarity problem, Computational Optimization and Applications (2016), DOI:10.1007/s10589016-9872-7

[15] X. Li AND M.K. NG, Solving sparse non-negative tensor equations: algorithms and applications, Frontiers of Mathematics in China, 10 (2015), pp. 649-680.

[16] L.-H. LiM, Singular values and eigenvalues of tensors: A variational approach, in IEEE CAMSAP 2005: First International Workshop on Computational Advances in Multi-Sensor Adaptive Processing, 2005, pp. 129-132.

[17] O.L. Mangasarian, Absolute value programming, Computational Optimization and Applications, 36 (2007), pp. 43-53.

[18] — Knapsack feasibility as an value equation solvable by successive linear programming, Optimization Letters, 3 (2009), pp. 161-170.

[19] O.L. Mangasarian and R.R. Meyer, Absolute value equations, Linear Algebra and its Applications, 419 (2006), pp. 359-367.

[20] R. Mifflin, Semismooth and semiconvex functions in constrained optimization, SIAM Journal on Control and Optimization, 15 (1977), pp. 959-972.

[21] L. QI, Eigenvalues of a real supersymmetric tensor, Journal of Symbolic Computation, 40 (2005), pp. 1302-1324.

[22] L. QI AND J. Sun, A nonsmooth version of Newton's method, Mathematical Programming, 58 (1993), pp. 353-367.

[23] L. QI AND H. Yin, A strongly semismooth integral function and its application, Computation Optimization and Applications, 25 (2003), pp. 223-246.

[24] L. QI, D. Sun, And G. Zhou, A new look at smoothing Newton methods for nonlinear complementarity problems and box constrained variational inequalities, Mathematical Programming, 87 (2000), pp. 1-35.

[25] Y. Song AND L. QI, Properties of some classes of structured tensors, Journal of Optimization Theory and Applications, 165 (2015), pp. 854-873.

[26] - Tensor complementarity problem and semi-positive tensors, Journal of Optimization Theory and Applications, 169 (2016), pp. 1069-1078.

[27] D. Sun AND J. Sun, Strong semismoothness of the Fischer-Burmeister SDC and SOC complementarity functions, Mathematical Programming, 103 (2005), pp. 575-581.

[28] D. Sun AND L. QI, On NCP-functions, Computation Optimization and Applications, 13 (1999), pp. 201-220.

[29] L. Zhang, L. QI, AND G. Zhou, M-tensors and some applications, SIAM Journal on Matrix Analysis and Applications, 35 (2014), pp. 437-452.

[30] G. Zhou, L. Caccetta, And K.L. Teo, A superlinearly convergent method for a class of complementarity problems with non-Lipschitzian functions, SIAM Journal on Optimization, 20 (2010), pp. 1811-1827. 